\title{
« Mon dire est un faire ». L'art de persuader dans les sermons politiques de Savonarole
}

\section{Cécile Terreaux-Scotto}

\section{OpenEdition \\ Journals}

Édition électronique

URL : http://journals.openedition.org/cei/254

DOI : $10.4000 /$ cei. 254

ISSN : 2260-779X

\section{Éditeur}

UGA Éditions/Université Grenoble Alpes

\section{Édition imprimée}

Date de publication : 15 avril 2005

Pagination : 89-117

ISBN : 978-2-84310-065-9

ISSN : $1770-9571$

Référence électronique

Cécile Terreaux-Scotto, « « Mon dire est un faire ». L'art de persuader dans les sermons politiques de Savonarole », Cahiers d'études italiennes [En ligne], 2 | 2005, mis en ligne le 15 octobre 2006, consulté le 26 mars 2021. URL : http://journals.openedition.org/cei/254 ; DOI : https://doi.org/10.4000/cei.254 


\title{
«MON DIRE EST UN FAIRE » \\ L'ART DE PERSUADER DANS LES SERMONS POLITIQUES \\ DE SAVONAROLE
}

\author{
Cécile Terreaux-Scotto \\ Université Stendhal Grenoble 3
}

En 1490, lorsque Savonarole commence à prêcher à Florence au couvent de San Marco, il reprend une prophétie qu'il avait exposée six ans plus tôt, en 1484, à San Gimignano : à l'issue d'une période de tribulations l'Église sera "flagellée et renouvelée " et cela ne saurait tarder. Dans ses premiers sermons sur Aggée qui débutent le $1^{\text {er }}$ novembre 1494, le prédicateur dominicain presse les Florentins de se repentir et de réformer leurs mœurs, seuls moyens d'échapper à la punition divine incarnée par le souverain français Charles VIII, qui a franchi les Alpes au début du mois de septembre. Mais au moment où les Florentins, face au vide institutionnel laissé par le départ forcé de Pierre de Médicis le 9 novembre 1494, s'interrogent sur la meilleure façon de gouverner dorénavant la cité, Savonarole intervient dans la vie politique. Il considère son auditoire non plus seulement comme une assemblée de fidèles qu'il faut amener à la conversion, mais aussi comme un groupe de citoyens qu'il faut aider à trouver un mode de gouvernement adapté à la nouvelle situation. Sous son impulsion, Florence se dote les 22 et 23 décembre 1494 de nouvelles institutions : le grand conseil et le conseil des quatre-vingts.

Toutefois, Savonarole estime que ces conseils ne fonctionneront pas correctement tant que les Florentins n'auront pas renoncé au "vieil usage » (l'enseignement reçu de leurs ancêtres) pour le remplacer par une " nouvelle coutume » (l'enseignement des premiers chrétiens). Il entreprend alors de persuader son auditoire de la nécessité de se convertir. En cela, il ne fait que remplir sa mission de prédicateur ; de plus, la prédication, « qui a pour fonction de persuader les destinataires " ${ }^{1}$, est un exercice codifié

1. Vittorio Coletti, L'éloquence de la chaire. Victoires et défaites du latin entre Moyen Âge et Renaissance, Paris, Cerf, 1987, p. 18. 
qui obéit à des règles. Mais Savonarole ne va pas chercher à respecter le code des Artes praedicandi ${ }^{2}$. Il accuse en effet les prédicateurs qui l'ont précédé de s'être comportés non pas comme des prêcheurs mais comme des poètes et des orateurs qui ont davantage travaillé la beauté formelle de leur discours qu'ils n’ont transmis la vérité. Pour émouvoir leur auditoire - au sens étymologique de " mettre en mouvement, pousser, déterminer " - les prédicateurs auraient donc jusque-là davantage cherché à plaire qu’à instruire.

Mon propos n'est pas de chercher à savoir si les accusations de Savonarole sont fondées ou non. Je n'entends pas non plus situer la prédication savonarolienne dans la tradition des Artes praedicandi. Je ne conduirai donc pas d'analyse stylistique des sermons ${ }^{3}$. Pour définir l'art de persuader chez Savonarole, j'étudierai le discours que le prédicateur tient sur ses propres sermons. Cela me conduira à examiner comment Savonarole expose, explique et justifie, à l'intérieur même de ses sermons, son emploi d'une langue que l'on peut qualifier de "nouvelle " à plusieurs titres. Nouvelle, parce que le prédicateur entend se distinguer de ses prédécesseurs. Nouvelle, parce que les contemporains la ressentent comme telle. Nouvelle enfin, parce qu'elle cherche à introduire cette "nouvelle coutume " que Savonarole présente comme une condition essentielle à la réforme.

Mon enquête s'appuiera essentiellement sur les sermons prononcés entre le $1^{\text {er }}$ novembre 1494 et le 18 mars $1498^{4}$. Mais j'exploiterai égale-

2. Joseph Schnitzer, Savonarola, Milan, Fratelli Treves, 1931, vol. 1, p. 232-249. Pour un point de vue plus nuancé, voir Marina MarietTI, "L'emploi des figures du discours dans la prédication de Savonarole ", in Savonarole. Enjeux, débats, questions. Actes du Colloque international (Paris, 25-2627 janvier 1996) éd. A. Fontes, J.-L. Fournel \& M. Plaisance, Paris, Université de la Sorbonne Nouvelle, 1997, p. 1-17 et en particulier p. 3-5. Sur les Artes praedicandi, voir Th.-M. CharLand o.p., Artes praedicandi. Contribution à l'histoire de la rhétorique au Moyen Âge, Paris-Ottawa, Vrin-Institut d'études médiévales, 1936, et Carlo Delconno, Giordano da Pisa e l'antica predicazione volgare, Florence, Olschki, 1975 , p. 83-237.

3. Pour une étude de ce type, nous renvoyons par exemple à M. Marietti, «L'emploi des figures du discours ", cit., et Philippe Guerin, "La petite clé des secrets". De la performativité du discours savonarolien ", in Savonarole. Enjeux, débats, questions, cit., p. 19-33.

4. Ces textes, publiés dans l'édition nationale des œeuvres de Savonarole parue chez Angelo Belardetti depuis 1955, nous sont connus grâce aux notes prises par le notaire Lorenzo Violi lorsqu'il écoutait le prédicateur. Les cycles des Prediche sopra Aggeo (1 ${ }^{\text {er }}$ novembre 1494-28 décembre 1494) et des Prediche sopra Giobbe ( $1^{\text {er }}$ mars 1495-24 avril 1495), édités à Venise respectivement en 1544 et en 1545, ont été établis par Violi à partir des autographes des schémas latins que Savonarole utilisait pour improviser ses sermons en vulgaire une fois en chaire. Les Prediche sopra i Salmi (6 janvier 1495 - 25 janvier 1495 puis 8 mai 1495 - 25 octobre 1495) et les Prediche sopra Ezechiele (30 novembre 1496 - 28 décembre 1496 puis 8 février 1497 - 20 mars 1497) ont été reconstituées grâce aux notes prises par Violi, notes qu'il a toutefois prises de façon peu précise, « in substantia " comme il l'indique lui-même. En revanche, ser Violi a intégralement transcrit les Prediche sopra Amos e Zaccaria (17 février 1496 - 10 avril 1496), les Prediche sopra Ruth e Michea (8 mai 1496 - 27 novembre 1496) et les Prediche sopra l'Esodo (11 février 1498 - 18 mars 1498), " de manière fidèle " selon l'appréciation de Savonarole. Il est admis que le 
ment deux des nombreux traités que le prédicateur écrivait ponctuellement et qu'il destinait à une publication rapide : l'Apologeticus de ratione poeticae artis, traité sur l'art poétique rédigé en 1491 en réponse au Carmen de Christianae Religionis ac Vitae Monasticae foelicitate écrit par l'humaniste Ugolino Verino, et le De Veritate prophetica dyalogus que Savonarole a rédigé en 1497 en réponse à l'excommunication qu'avait lancée contre lui le pape Alexandre VI le 13 mai de la même année.

Dès le sermon XXI sur Aggée, prononcé le 25 décembre 1494, Savonarole proclame la supériorité de l'Écriture sainte sur la rhétorique cicéronienne :

Dicano questi dotti che la rettorica di Cicerone è una efficace dottrina e che la muove assai. Questi non considerano quanto più vale la dottrina di Cristo. Tre cose fa la rettorica : illumina, deletta e inclina. E sebbene la retorica di Tullio insegna o fa questi effetti, molto più gli fa la Scrittura sacra. ${ }^{5}$

Il n'y a jusque-là rien d'exceptionnel à ce qu'un homme d'Église revendique l'auctoritas des Saintes Écritures en leur attribuant les trois ressorts de l'éloquence persuasive définis dans l'Orateur : «instruire, émouvoir et plaire ${ }^{6}$.

Mais ce qui se joue en réalité chez Savonarole, c'est la définition d'un nouvel art de persuader, où la vérité l'emporte sur la beauté, la "simplicité " sur les artifices esthétiques, l'action sur la parole, où la persuasion passe davantage par l'instruction que par la séduction.

C'est pourquoi frère Jérôme va s'adresser aux Florentins comme un pédagogue. Comme les Florentins doivent être éduqués dans le sens du projet voulu par Dieu, l'art de persuader devient d'abord l'art d'expliquer et de montrer par l'exemple, à travers un discours rendu accessible à tous.

C'est qu'en ces temps troublés des guerres d'Italie, où il faut faire face à un état d'urgence permanent et à la "diversité des temps ", Savonarole doit trouver une langue qui persuade les Florentins d'agir - et le plus rapidement possible - pour la mise en ouvre de la réforme de leur cité, avant que les fléaux ne s'abattent sur elle.

prédicateur a relu la plupart des textes qui sont parus de son vivant. Sur le travail accompli par Lorenzo Violi et la fiabilité de ses transcriptions, voir Gian Carlo GarfaGninI, "Ser Lorenzo Violi e le prediche del Savonarola ", in "Questa è la terra tua ". Savonarola a Firenze, Florence, Sismel - Edizioni del Galluzzo, 2000, p. 3-27 et Lorenzo Violi, Le Giornate, a cura di G.C. Garfagnini, Florence, Olschki, 1986.

5. Girolamo Savonarola, Prediche sopra Aggeo - Trattato circa el reggimento e governo della città di Firenze, a cura di Luigi Firpo, Rome, Angelo Belardetti, 1965, XXI, p. 381 : «Les savants disent que la rhétorique de Cicéron est un savoir efficace et qu'il ébranle beaucoup. Ils ne considèrent pas à quel point le savoir du Christ a davantage de valeur. La rhétorique fait trois choses : elle instruit, elle plaît et elle émeut. Et bien que la rhétorique de Cicéron enseigne ou produise ces effets-là, l'Écriture sainte les produit bien davantage ".

6. L'orateur XXI, 69 (Cicéron, L'orateur, texte établi et traduit par Albert Yon, Paris, Les Belles Lettres, 1964). 


\section{Cécile Terreaux-Scotto}

Confessiamo, dico, confessiamo el nostro errore : noi predicavamo tuttodì retorica e poesia, ed era diventato notte, e però non pigliammo pesce alcuno. Non si faceva quasi conto della fede, e però savamo al buio.

C'est en ces termes que, le 6 avril 1496, Savonarole décrit la situation dans laquelle Florence se trouvait avant qu'il ne monte en chaire. Cependant, ce que condamne Savonarole, c'est peut-être moins la poésie en tant que telle - il a lui-même composé des textes en vers ${ }^{8}$ - que l'usage qui en est fait par les prédicateurs, comme le suggère l'ouverture de l'Apologeticus de ratione poeticae artis :

nec ego aliquando artem poeticam damnandam putavi, sed quorundam abusum [...] Mea igitur intentio est quid de arte poetica sentiam in hoc libello describere, et adversus quosdam sciolos stilum dirigere qui in modico atque vano ne dicam iuvenili verborum amore falluntur.?

Certes, dans ses sermons Savonarole préconisera l'abandon dans les écoles de l'étude de certains textes au profit de livres jugés plus sains. Le 19 février 1496, il insiste ainsi pour que

|...] non si leggessi per le scuole poeti cattivi, come è Ovidio De arte amandi, Tibullo, né Catullo e simili, né Terenzio dove parla di quelle meretricule. Leggete san Ieronimo, santo Augustino e altri libri ecclesiastici, overo Tullio, Virgilio e qualche cosa di Scrittura Santa. E dove voi, maestri, trovate in quelli vostri libri di poesie Iove, Plutone, ecc., dite loro : - Figliuoli miei, queste sono favole -, e mostrateli che solo Dio è quello che regge el mondo. ${ }^{10}$

Quelques jours plus tard, le 7 mars, il va même jusqu’à réclamer la mise en place d'une législation :

[...] ma si vorria fare una legge che 'l fussi escluso Ovidio De arte amandi, la Priapea e certi altri libri che non bisogna dirgli qua, e' quali insegnano mille lascivie ; tollerarei Vergilio, Omero in greco, Tullio, e mescolare' una lezione di santo Agostino De civitate Dei, di santo Ieronimo o qualche altra cosa di Scrittura Santa ; e che non

7. G. Savonarola, Prediche sopra Amos e Zaccaria, a cura di Paolo Ghiglieri, Rome, Angelo Belardetti, 1969, XLVII, vol. 3, p. 363 : «Avouons, dis-je, avouons notre erreur : nous ne prêchions toute la journée que rhétorique et poésie, et la nuit était tombée et c'est pourquoi nous ne prîmes aucun poisson. On ne se souciait presque pas de la foi, aussi étions-nous dans l'obscurité ".

8. Cf. G. Savonarola, Poesie, a cura di Mario Martelli, Rome, Angelo Belardetti, 1968.

9. G. Savonarole, Apologeticus de ratione poeticae artis in Scritti filosofici, a cura di G.C. Garfagnini \& Eugenio Garin, Rome, Angelo Belardetti, 1982, vol. 1, p. 211-212 ; traduction d'après Jérôme Savonarole, La fonction de la poésie, traduit et annoté par Bruno Pinchard [précédé de Bruno Pinchard, Le noud de la colère], Lausanne, Éditions l'Âge d'Homme, 1989, p. 109: " pas une fois je n'ai pensé condamner l'art poétique, mais l'abus de certains [...] Mon intention est de décrire dans ce petit livre ce que je pense de l'art poétique, et de diriger la pointe de mon style contre certains demi-habiles qui sont tombés dans un amour sans grandeur et vain, pour ne pas dire infantile, pour les mots".

10. Prediche sopra Amos e Zaccaria, III, éd. cit., vol. 1, p. 92 : « [...] dans les écoles il ne faut pas lire les poètes mauvais, comme l'est Ovide avec son De arte amandi, Tibulle, ni Catulle et d'autres semblables, ni Térence quand il parle de ces petites prostituées. Lisez saint Jérôme, saint Augustin et d'autres livres ecclésiastiques, ou alors Cicéron, Virgile et quelques textes des Saintes Écritures. Et là où vous, les maîtres, vous trouvez dans vos livres de poésie Jupiter, Pluton, et cætera, dites-leur : - Mes enfants, ce sont des contes -, et montrez-leur que Dieu seul gouverne le monde ». 
abbino mai una lezione de' pagani che non abbiano anche una de' cristiani. Sappiate, fanciugli miei, che questi dei, come è Giove, Plutone e gli altri che voi trovate sopra a quelli libri, furon tutti cattivi uomini, o le son favole. ${ }^{11^{2}}$

Mais déjà au début du Xve siècle, Giovanni Dominici écartait, avec plus de sévérité encore puisqu'il n'acceptait aucune lecture qui ne soit pas chrétienne, les textes qui selon lui sont " più insegnanti d'amare secondo carne che mostratori di buon costumi " ${ }^{12}$. Et les enfants "prima diventando pagani che cristiani, e prima chiamando dio Iuppiter o Saturno, Venus o Cibeles, che il sommo Padre, Figliuolo e Spirito santo " ${ }^{13}$, il avait rédigé en 1405 la Lucula noctis pour apporter la lumière de la foi dans la nuit du paganisme réintroduit par les humanistes. Par ce traité, Dominici entendait répondre à Coluccio Salutati. Ce dernier avait en effet affirmé, contre le moine Giovanni da San Miniato qui considérait la poésie comme une activité inutile et nocive, que non seulement Dieu avait utilisé la poésie à travers les psalmistes, mais que la Bible elle-même était remplie de choses détestables : le péché d'Ève et de Caïn, les vices des Sodomites, les méfaits des frères de Joseph, sans compter le Cantique des Cantiques, texte des plus lascifs ${ }^{14}$.

Savonarole s'inscrit donc dans la polémique que des hommes d'Église mènent contre les poètes et les humanistes lorsqu'il affirme que l' " obscurité » enveloppe Florence parce que les prédicateurs, " ayant perdu le feu de l'Esprit Saint », ont délaissé les Saintes Écritures au profit de la poésie, accordant plus d'importance au paganisme qu'au christianisme. Il affirme à ce propos, le 18 ou 19 décembre 1494 (la date du sermon est incertaine), puis de nombreuses fois par la suite, que

[...] su' pergami non si predicava Cristo, ma eranvi introdotte le poesie e Dante e cose frivole : e la Scrittura sacra si restava là nella polvere : e li poeti andavano su per

11. Prediche sopra Amos e Zaccaria, XX, éd. cit., vol. 2, p. $81:$ : [...] il faudrait faire une loi qui exclue Ovide et son De arte amandi, la Priapea et certains autres livres qu'il ne faut pas citer ici, qui enseignent mille choses lascives; je tolérerais Virgile, Homère en grec, Cicéron, et j’y ajouterais une lecture du $D e$ civitate Dei de saint Augustin, de saint Jérôme ou de quelques autres textes des Saintes Écritures. Et il faudrait que [les enfants] ne reçoivent jamais une leçon des païens sans en recevoir également une des chrétiens. Sachez, mes enfants, que ces dieux, tels que Jupiter, Pluton et les autres que vous rencontrez dans ces livres, ont tous été des hommes mauvais, ou bien que ce sont des contes ".

12. Giovanni Dominici, Regola del governo di cura familiare, a cura di Donato Salvi, Florence, Angiolo Garinei, 1860, p. 135 : " enseignent davantage à aimer selon la chair qu'ils ne montrent les bonnes mœurs ".

13. Ibid., p. 135 : « devenant d'abord païens avant d'être chrétiens, et appelant dieu d'abord Jupiter ou Saturne, Vénus ou Cybèle, plutôt que le très haut Père, le Fils et le Saint Esprit ».

14. Antonio Lanza, Polemiche e berte letterarie nella Firenze del primo Rinascimento (1375-1449), seconda edizione completamente rifatta, Rome, Bulzoni, 1989, p. 81-106. Sur la Lucula noctis, voir Giuseppe SaItTA, L'educazione dell'umanesimo in Italia, Venise, La Nuova Italia Editrice, 1928, p. 122128. 
li pergami, e’ quali poeti già condussono la idolatria ed el paganesimo per tutto el mondo. ${ }^{15}$

D'après Savonarole, les poètes « non hanno altro ch'el suono de'loro versi ${ }^{16}$. «Impleverunt chartas superbissimis obscuritatibus vanissimisque verborum ornatibus " ${ }^{17}$, et par conséquent " relinquit in cortice litterae $"{ }^{18}$. Autrement dit, ils ne se préoccupent que de l'enveloppe de leur discours et non de sa véracité ni de son efficacité. Savonarole condamne donc l'aspect rhétorique de la poésie. En se comportant comme les poètes, qui portent un amour excessif aux mots, les prédicateurs ont confondu discours de séduction et discours de persuasion. De l'art de persuader, ils n'ont retenu que le plaisir à donner à l'auditoire. À cet égard, il est savoureux que le seul poète que Savonarole cite ici nommément soit Dante ${ }^{19}$, lui qui, fustigeant les " ciance " (sottises) des prédicateurs de son temps, déplorait qu' " ora si va con motti e con iscede / a predicare, e pur che ben si rida, / gonfia il cappuccio, e più non si richiede " ${ }^{20}$. Car c'est bien une accusation semblable que formule Savonarole quand il estime que les prédicateurs qui l'ont précédé ont oublié d'instruire les Florentins et n'ont pas su les émouvoir, c'est-à-dire les pousser à agir dans le sens exigé par la foi.

Aussi frère Jérôme entend-il ne pas se soucier en premier lieu de la forme de ses sermons pour s'occuper exclusivement de convaincre les Florentins d'emprunter "la via de' santi " ${ }^{21}$, et ce, dans un but politique. Selon lui en effet la réforme politique de la cité ne pourra avoir lieu que si

15. Prediche sopra Aggeo, XVII, éd. cit., p. 290 : «[...] en chaire on ne prêchait pas le Christ, mais on avait introduit les poésies et Dante et des choses frivoles; et l'Écriture sainte restait là dans la poussière ; et les poètes montaient en chaire, eux qui ont répandu l'idolâtrie et le paganisme dans le monde entier "; voir aussi ibid., XI, p. 187 ; G. Savonarola, Prediche sopra Giobbe, a cura di Roberto Ridolfi, Rome, Angelo Belardetti, 1957, XXVI, vol. 2, p. 44 ; XXXII, vol. 2, p. 125 et p. 131 ; XLII, vol. 2, p. 333-334; ID., Prediche sopra Amos e Zaccaria, XII, éd. cit., vol. 1, p. 313 et p. 336-337 ; XLI, vol. 3, p. 170-171 ; ID., Prediche sopra Ruth e Michea, a cura di Vincenzo Romano, Rome, Angelo Belardetti, 1962, IV, vol. 1, p. 120 ; XVIII, vol. 2, p. 88 ; ID., Prediche sopra Ezechiele, a cura di R. Ridolfi, Rome, Angelo Belardetti, 1955, XII, vol. 1, p. 165.

16. Prediche sopra Aggeo, XI, éd. cit., p. 182 : "n'ont pas autre chose que le son de leurs vers ».

17. Apologeticus, cit., p. 242 : «Ils couvrent le papier d'obscurités prétentieuses et de vaines figures de style " (La fonction de la poésie, cit., p. 136).

18. Apologeticus, cit., p. 255 : "ils en restent à l'écorce de la lettre " (La fonction de la poésie, cit., p. 146).

19. Ailleurs, Savonarole appelle à brûler le Morgante, écrit par Luigi Pulci à la demande de Lucrezia Tornabuoni, la mère de Laurent le Magnifique. Voir Prediche sopra Aggeo, cit., I, p. 20 et infra n. 93. Dans les sermons il fait également allusion aux Triomphes de Pétrarque, pour déplorer que les femmes en possèdent des exemplaires ornés et il raconte à ce propos qu’on lui en a montré une fois un exemplaire qui valait plus de cinquante ducats ; cf. Prediche sopra Amos e Zaccaria, XLI, éd. cit., vol. 3, p. 170-171.

20. Paradiso XXIX, v. 115-117: "On prêche à présent avec des facéties/et des quolibets, et pourvu qu'on rie bien,/le capuchon se gonfle, et ne demande rien ". Nous empruntons la traduction de Jacqueline Risset, éditée chez Flammarion en 1990.

21. Prediche sopra Aggeo, II, éd. cit., p. 35 : « le chemin des saints ». 
les Florentins acceptent de se convertir : les « bons » citoyens sont d'abord de «bons " chrétiens qui ont la foi, acceptent de vivre dans le respect des commandements de Dieu et mènent une vie de prière. Ainsi, voulant persuader les Florentins que la réforme politique est un acte de foi en Dieu qui ouvre le chemin du salut, Savonarole les exhorte inlassablement à venir écouter son prêche puisque

[...] la fede viene da l'udire, da l'udire, dico, el verbo di Cristo. Imparono adunque li òmini la fede per la lingua del predicatore, e è necessario udire Dio per la lingua del predicante. ${ }^{22}$

Certes, transmettre l'enseignement de Dieu fait partie de la mission traditionnelle des prédicateurs, " col latte de' quali e colla loro dottrina si debbono nutrire e'cristiani, per condurli a perfezione " ${ }^{23}$. De la même façon, insister sur l'utilité des sermons relève des topö̈ de la prédication ${ }^{24}$. Mais si frère Jérôme demande aux Florentins de venir l'écouter, c'est parce qu'il compte les convaincre de la justesse de ses propos et les persuader de mettre en place la réforme religieuse, morale et, plus encore, la réforme politique qu'il présente au cours de ses sermons. Or, pour se différencier des prédicateurs qui l'ont précédé, il va compter davantage sur la rigueur pédagogique que sur l'attrait esthétique de son prêche. Loin d'enrober son texte dans des artifices rhétoriques, Savonarole le "déplie » pour le rendre accessible aux Florentins.

Il ne proscrit pas pour autant les figures de style qu'il juge " vaines » chez les poètes. Seulement, il ne les utilise pas comme ornement du discours, puisqu'il considère qu'un discours peut être persuasif sans avoir besoin de séduire, dès lors qu'il instruit. Ne cherchant pas à plaire mais à se faire comprendre, Savonarole prend donc le soin d'expliquer chacune des métaphores et des allégories qu'il utilise afin que les Florentins en comprennent le sens puis mettent en pratique l'enseignement qu'ils en retirent.

Ainsi, les allégories tirées de la Bible doivent expliciter la parole de Dieu pour convaincre l'auditoire de la vérité de ce qui est dit et le persuader du même coup de se "renouveler ». Ce n'est pas un hasard si l'allégorie fait

22. Prediche sopra Ruth e Michea, XII, éd. cit., vol. 1, p. 346 : «[...] la foi provient de l'écoute, de l'écoute, dis-je, du verbe du Christ. Les hommes apprennent donc la foi par la bouche du prédicateur, et il est nécessaire d'écouter Dieu par la bouche du prêcheur ".

23. Prediche sopra Giobbe, IX, éd. cit., vol. 1, p. 164-165 : « [...] doivent nourrir les chrétiens avec leur lait et leur savoir pour les faire parvenir à la perfection ".

24. Pour ne donner qu'un seul exemple, Bernardin de Sienne rappelait fréquemment à son auditoire tout le profit qu'il pourrait tirer de son prêche : l'enseignement qu'il y recevrait servirait au salut spirituel de la cité; voir Bernardino da Siena, Le prediche volgari, a cura di Piero Bargellini, Milan-Rome, Rizzoli, 1936, III, p. 81 et p. 89 ; XI, p. 252. 
partie du discours prophétique de frère Jérôme. Ce dernier insiste en effet sur le fait que l'allégorie doit dire des choses réelles et non fictives comme lorsqu'elle est utilisée par les poètes. Ces choses réelles sont destinées à annoncer le futur, ce dont seul Dieu est capable. Parce que l'allégorie est un moyen employé pour instruire sur l'avenir et non pour plaire, son contenu doit être rendu accessible. C'est pourquoi, à chaque fois que Savonarole rappelle, conformément à la tradition médiévale, que les Saintes Écritures possèdent quatre sens (littéral, allégorique, moral et anagogique) ${ }^{25}$, il ne manque pas d'expliciter le fonctionnement de chacun de ces sens, scandant ses explications par des formules récurrentes du type " cela signifie » (significa), " cela veut dire " (vuol dire). Un passage du sermon XI sur Job est emblématique à cet égard :

E come uno nome posto dall'uomo alle cose ha diversi significati, molto maggiormente Iddio, che ha la plenitudine della potestà, ha potuto fare che nella sua Sacra Scrittura siano diversi sensi, e che uno medesimo parlare abbia diversi intelletti, e una medesima parola abbia diversi significati. Come, verbigrazia, quando la Scrittura dice: Lauda Ierusalem Dominum, vuol dire quella città materiale di Ierusalem, e significa ancora la Chiesa militante e ancora la trionfante, dove si lauda il Signore, e pigliasi ancora per l'anima che lauda Dio. ${ }^{26}$

Dans le sermon XVIII sur les Psaumes, qui raconte le transport de l'arche de Moïse par David de Gat à Jérusalem, Savonarole explique en ces termes pourquoi il a exposé les quatre significations de l'Écriture de Dieu :

Questo discorso ti ho fatto, perché io ti voglio questa mattina introdurre la Scrittura della traslazione dell'arca di Moisè, quando David la portò di Gabaa in Ierusalem, al proposito della processione della Vergine che vogliamo fare nel portare la tavola di santa Maria in Pruneta qua domani. ${ }^{29}$

Frère Jérôme entend préparer la cérémonie célébrative du lendemain. Pour que les Florentins y participent avec le recueillement, l'humilité et le dévouement à Dieu nécessaires - et qu’ils montrent de cette façon leur désir de participer à la réforme de la cité voulue par Dieu -, ils doivent connaître l'épisode biblique et surtout l'interprétation allégorique qui en est faite.

25. Voir par exemple G. Savonarola, Prediche sopra i Salmi, a cura di Vincenzo Romano, Rome, Angelo Belardetti, 1969, III, vol. 1, p. 46.

26. Prediche sopra Giobbe, XI, éd. cit., p. 187 : «Et de la même façon qu'un nom donné par l'homme aux choses a plusieurs sens, Dieu, bien plus encore, qui a la plénitude de la puissance, a pu faire en sorte que dans son Écriture sainte se trouvent plusieurs sens, et qu'un même langage ait plusieurs acceptions, et qu'un même mot ait plusieurs significations. Comme, par exemple, quand les Écritures disent : Lauda Ierusalem Dominum, cela veut dire la ville matérielle de Jérusalem, et cela signifie aussi l'Église militante et également l'Église triomphante, où on loue le Seigneur, et on peut aussi la considérer comme l'âme qui loue Dieu "; voir aussi ibid., XXVI, vol. 2, p. 44 ; XLII, vol. 2, p. 333.

27. Ibid, XVIII, éd. cit., vol. 1, p. 289 : " J'ai fait ce discours parce que ce matin je veux introduire l'Écriture du transport de l'arche de Moïse, quand David la porta de Gat à Jérusalem, en lien avec la procession de la Vierge que nous voulons faire demain en apportant ici le tableau de sainte Marie de Pruneta». 
L'arche signifie à la fois le Christ, l'Église et la Vierge. Mais Savonarole choisit de ne parler que de la Vierge, puisque la procession du lendemain lui est consacrée. L'arche exprime alors la pureté, la gloire, la charité et la dignité virginales. Elle repose sur un char qui représente "il novo vivere » de Florence. Les Florentins sont entraînés vers cette " nouvelle vie " par "les prédicateurs ", représentés par les bœufs qui tirent le char. Cet attelage est aiguillonné par un fouet (virga) qui figure la puissance que la Vierge a reçue de Dieu pour apporter des grâces à Florence.

Pédagogue, le prédicateur décortique donc le texte biblique pour le mettre à la portée de son auditoire. De la même façon que l'arche de Moïse avait été transportée de Gat à la ville sainte, le portrait de Marie sera transporté à Florence, dont le prédicateur veut précisément faire une «nouvelle Jérusalem ». Le discours de persuasion pédagogique, articulé autour d'une allégorie biblique, sert ainsi de support à la mise en place de la réforme et de moment préparatoire à l'action ${ }^{28}$. En définitive, tout se passe comme si Savonarole utilisait une allégorie tirée de la Bible pour persuader les Florentins du bien fondé de son propre discours et pour les inciter à agir pour "sa" réforme.

De ce point de vue, l'utilisation qu'il fait des métaphores filées est très significative. Lorsqu'il file la métaphore des « enfants de cent ans » de 1494 à 1498, Savonarole expose son point de vue sur la réforme politique en montrant aux Florentins la façon dont ils doivent se convertir. Jusqu'au 17 février 1496, la métaphore a deux sens. Premièrement, elle signifie que les Florentins refusent délibérément de se tourner vers Dieu mais qu'ils peuvent encore se convertir et être éduqués. Il s'agit alors pour Savonarole de leur faire comprendre qu'il est temps pour eux de faire pénitence et d'adhérer à la réforme. Deuxièmement, la comparaison des Florentins à des enfants conduit Savonarole à évoquer l'enfance spirituelle, cette attitude de simplicité qui ouvre le Royaume des Cieux et qu'il recommande à son auditoire d'adopter. Puis, le 17 février 1496, au lendemain de la première procession des fanciulli dans la cité, qui marque le début de leur participation à la mise en place de la réforme morale de la cité, la métaphore prend un autre sens. Les fanciulli florentins ont adopté une attitude que Savonarole juge digne de l'enfance spirituelle. Si bien qu'ils font désormais coïncider l'enfance réelle, entendue en tant que classe d'âge, avec l'enfance métaphorique, qui désigne l'attitude de simplicité ouvrant le Royaume des Cieux. Savonarole peut dès lors s'appuyer sur l'image qu'il

28. Nous verrons que c'est précisément là un des aspects fondamentaux de la prédication savonarolienne. 
a développée au cours de ses sermons pour tenter d'inculquer aux Florentins une idée qui allait à l'encontre même de leur organisation familiale et sociale : la nécessité où ils se trouvent d'imiter leurs enfants, qui seront leurs guides sur le chemin de la réforme ${ }^{29}$. En déployant toutes les significations d'une seule formule - les « enfants de cent ans » - Savonarole met donc en place un discours pédagogique. L'explicitation de la métaphore lui permet d'aboutir à une proposition qui lui est progressivement apparue comme essentielle à son projet : dès lors que les fanciulli devenaient ses partisans, tandis qu'une partie des adultes refusait de le suivre, l'inversion de l'ordre traditionnel des générations s'imposait, comme une condition nécessaire à la réforme de Florence.

Ainsi, Savonarole utilise les artifices de l'éloquence pour expliquer le sens de sa prédication, parce que c'est de ce sens que doit naître la réforme. C'est que d'après lui, les ornements rhétoriques employés sans visée pédagogique ne sont que des figures de séduction qui n'ont pas leur place dans un discours de persuasion : "il predicar a pompa è cosa che solo diletti l'orecchie, e non muova il cuor dell'uomo " ${ }^{30}$, affirme-t-il significativement. Chez lui, l'art de persuader ne passe donc pas par la beauté des figures du discours mais par l'explication du sens de ces mêmes figures.

Aussi le prédicateur est-il séduit par «l'éloquence» des Saintes Écritures, qui n'ont justement pas besoin d'artifices " ad inclinandum hominum animos firmandosque in bonis moribus " ${ }^{31}$, parce qu'elles possèdent en elles-mêmes la capacité de persuader, contrairement à la rhétorique cicéronienne par exemple.

Dans le sermon XXI sur Aggée, Savonarole explique à ce propos qu'alors que l'éloquence théorisée par les Anciens cherche à convaincre en faisant appel à la " lumière naturelle " (lume naturale) - la raison -, les Saintes Écritures possèdent la "lumière surnaturelle " (lume sopranaturale) - la grâce donnée par la foi ${ }^{32}$. Or, seule la « lumière surnaturelle " peut conduire les Florentins à vivre en chrétiens. Seule la foi peut leur faire retrouver la "forme naturelle " (l'état privilégié dans lequel vivaient les patriarches bibliques et les premiers chrétiens) perdue à cause de la

29. Pour une étude exhaustive de cette métaphore, voir Cécile Terreaux-Scotto, "Vous êtes des enfants de cent ans". Âge réel et âge métaphorique dans les sermons politiques de Savonarole " in Il Pensiero Politico, 2004.

30. Prediche sopra Giobbe, XXVI, éd. cit., vol. 2, p. 29 : "Prêcher avec pompe est une chose qui plaît seulement aux oreilles et n'émeut pas le cœur de l'homme "; cf. Prediche sopra Ruth e Michea, V, éd. cit., vol. 1, p. 142-143.

31. Apologeticus, cit., p. 255 : "plier les âmes et les confirmer dans les bonnes mœurs " (La fonction de la poésie, cit., p. 146).

32. Prediche sopra Aggeo, cit., XXI, p. 381. 
« seconde forme » (l'éducation) que l'Histoire leur a inculquée à travers la coutume $^{33}$. Et puisque « il lume della fede e il lume della Scrittura Sacra sono conformi e connaturali insieme » ${ }^{34}$, l'Écriture sainte "instruit » (illumina) les Florentins beaucoup plus que l'éloquence classique ne peut le faire ${ }^{35}$.

De plus, continue frère Jérôme, la « lumière surnaturelle » de l'Écriture sainte a incité les apôtres à tout quitter, les honneurs, les richesses et la vie (gli onori, la robba e la vita), ce que la "lumière naturelle " des orateurs n'est jamais parvenue à faire. Parce qu'elle parvient à stimuler les passions, l'Écriture sainte émeut (inclina) davantage que la rhétorique classique ${ }^{36}$.

Enfin, Savonarole déclare que « la bellezza esteriore viene dalla forma interiore della cosa " ${ }^{37}$. Étant donné que l'Écriture sainte provient de Dieu $^{38}$, la beauté de son contenu ne peut qu'irradier sa forme. Elle plaît (deletta) alors plus que l'éloquence cicéronienne.

Dans cette confrontation entre la rhétorique de Cicéron et l'éloquence des Saintes Écritures se dessine l'opposition entre la beauté et la vérité. Car pour Savonarole, l'emploi des " vaines figures de style " non seulement rend le discours inefficace mais fait des poètes, comme des orateurs, des menteurs : la beauté du langage confere au discours un enrobage factice, qui exalte faussement son contenu véritable. "Le colonne che paiono di porfido e sono di legno è la dottrina de'poeti, degli oratori ", disait-il à ce propos $^{39}$. En proclamant la supériorité des Saintes Écritures sur celle de la rhétorique cicéronienne, Savonarole proclame donc la supériorité de la vérité sur la beauté dans l'art de persuader. Il manifeste en même temps que l'instruction est plus importante que la séduction, raison pour laquelle il s'adresse aux Florentins non pas comme un poète ou comme un orateur mais avant tout comme un pédagogue.

Au modèle légué par les orateurs et les poètes Savonarole préfere alors celui des prophètes, dont les « chants » « et sapientiam et amorem divinum

33. Sur la " forme naturelle " et la " coutume ", voir John G.A. Pocock, Le moment machiavélien, Paris, PUF, 1997, p. 119-125.

34. Prediche sopra Giobbe: «[...] la lumière de la foi et la lumière de l'Écriture sainte sont identiques et ont aussi la même nature ", X, éd. cit., vol. 1, p. 168.

35. Prediche sopra Aggeo, XXI, éd. cit., p. 381.

36. Ibid., XXI, p. 381.

37. Ibid., XXI, p. 382 : " la beauté extérieure vient de la forme intérieure de la chose ».

38. Prediche sopra Giobbe, IX, éd. cit., vol. 1, p. 153 : "Così nella Scrittura, li profeti e altri che l'hanno scritta, sono stati l'istrumento, ma Dio è stato il principale agente, e però quella è perfetta e senza errore".

39. Scelta di prediche e scritti di fra Girolamo Savonarola con nuovi documenti intorno alla sua vita, a cura di P. Villari - E. Casanova, Florence, Sansoni, 1898, p. 47 : "Les colonnes qui paraissent de porphyre et qui sont de bois, c'est le savoir des poètes et des orateurs ". 
sanctamque redolent humilitatem ; non enim magnum studium verbis componendis, sed veritati dicendx adhibuerunt " ${ }^{40}$, et celui des apôtres qui « più hanno inclinato e più hanno possuto [...] con la nuda e semplice verità, che gli oratori con le loro ornate parole e con le loro orazioni piene di eloquenzia $"{ }^{41}$.

Mais l'emploi de la langue des prophètes et des apôtres ne permet pas seulement le triomphe de la foi et de la vérité sur la superficialité de la beauté. Cette langue, qui est celle de la "simplicité ", permet aussi d'indiquer aux Florentins l'exemple de la façon dont ils doivent se réformer.

Alors que les poètes placent "l'écorce avant la lettre », les fioritures avant le contenu du discours, les prédicateurs qui exposent les Saintes Écritures vont droit à la " lettre ». C'est que l'Écriture sainte, provenant de Dieu, expose la vérité de la foi. Mieux encore, elle est elle-même vérité : " questa Scrittura è da Dio [...] questa scrittura è più vera d'alcuna altra " ${ }^{42}$. La parole du prédicateur n'a pas dès lors à se montrer persuasive, elle est la persuasion même. Aussi peut-il se laisser porter par ses pensées, comme il l'explique lui-même :

Testis etenim mihi est Deus, quod non cogitem que proferam verba, nec quos gestus modosve observem : sed solum sententiis intentus, quo me trahit illarum incitatio et fervor spiritus, illuc simpliciter feror. ${ }^{43}$

Savonarole déclare négliger les recommandations de la rhétorique antique qui indiquaient la façon de trouver les bons arguments (inventio), les moyens d'employer des paroles efficaces pour persuader un auditoire (elocutio) et la codification des gestes accompagnant le discours persuasif de l'orateur (actio) ${ }^{44}$ parce qu'il veut faire de sa parole un instrument au service de la vérité et non une fin en soi au service de la beauté. Mais il y a plus. En effet, ce n'est pas un hasard s'il décrit le processus psychologique,

40. Apologeticus, cit., p. 254 : « [...] respirent la sagesse, l'amour divin et l'humilité sainte parce qu'ils n'usent pas d'une grande recherche dans l'arrangement des mots, mais dans l'énonciation du vrai " ( $\mathrm{La}$ fonction de la poésie, cit., p. 145).

41. Prediche sopra i Salmi, VI, éd. cit., vol. I, p. 102-103: «ont davantage ému et pu faire [...] avec la vérité nue et simple que les orateurs avec leurs paroles ornées et leurs discours pleins d'éloquence ".

42. Ibid., IV, p. 72 : " «cette Écriture vient de Dieu [...] cette Écriture est plus vraie qu'aucune autre" .

43. G. Savonarole, Verità della profezia. De veritate prophetica dyalogus, a cura di Claudio Leonardi, traduzione di Oddo Bucci, Florence, Sismel - Edizioni del Galluzzo, 1997, V, p. 99 : " Mi è testimone Dio, infatti, che io non penso alle parole che dico, né controllo la misura dei gesti, ma sono solo attento a seguire il mio pensiero e mi lascio semplicemente condurre là dove mi attira il movimento interno dei miei ragionamenti e il fervore dello spirito ".

44. CicÉron, L'orateur, cit., XVII, 59. 
intellectuel et spirituel qui oriente son discours dans son De veritate prophetica dyalogus. Car s'il affirme parler en même temps qu'il pense tout comme il pense en même temps qu'il parle, c'est parce qu'il ne se considère pas seulement comme un prédicateur : il est certain d'être aussi un prophète ${ }^{45}$.

Dieu, qui connaît l'éternité et qui seul connaît le moment où s'accomplira la réforme à Florence, a choisi de révéler ce moment à Savonarole, ce "pauvre frère " (fraticello) de Ferrare. Personne élue pour annoncer le futur, le prédicateur devient prophète, seul habilité à annoncer les événements à venir. Frère Jérôme précise toutefois que «Iddio non vuole che si predichi il tempo determinato " ${ }^{46}$ afin que les Florentins vivent toujours dans la crainte de ce qui pourrait advenir et soient constamment prêts à la pénitence. La seule indication chronologique précise concernant le temps de la réforme que fournit Savonarole concerne par conséquent la proximité des châtiments divins qui vont s'abattre sur la cité " rapidement " (cito).

Le prédicateur n'est alors qu'un instrument entre les mains du Seigneur. Dieu a choisi un religieux comme lui, sans connaissance ni puissance temporelle, rempli de péchés et étranger à Florence parce qu'il est le seul maître de la réforme de la citée ${ }^{47}$. La voix de Savonarole devient dès lors la voix humaine de Dieu qui communique, à travers lui, avec les Florentins : " com'ho detto più volte, Cristo è quello che prèdica e non io ", résumet-il le 13 décembre $1496^{48}$.

D’abord " annonciateur de la parole qui lui a été confiée ", comme le formule Philippe Guérin ${ }^{49}$, le prédicateur-prophète finit par s’identifier

45. Sur la conviction de Savonarole d'être un prophète, voir Jean-Louis Fournel - Jean-Claude ZaNCARINI, "Les temps de la prophétie ", in ID., La politique de l'expérience. Savonarole, Guicciardini et le républicanisme florentin, Alessandria, Edizioni dell'Orso, 2002, p. 41-53 ; ID., "Le procès de Savonarole : manipulation ou bilan ", in ibid., en particulier p. 88-97; ID., " Prophétisme et doutes de l'histoire : lectures contemporaines des procès ", in ibid., p. 99-116; G.C. GARFAGNINI, "Savonarola e la profezia : tra mito e storia ", in Studi Medievali, XXIX, 1988, p. 173-201 ; Armando Verde, "Girolamo Savonarola : ideologo e profeta. Il quaresimale del 1491 ", in Savonarola. Democrazia tirannide profezia, a cura di G.C. Garfagnini, Florence, Sismel-Edizioni del Galluzzo, 1988, p. 127-147 ; Claudio Leonardi, «Jérôme Savonarole et le statut de la prophétie dans l'Église ", in Les textes prophétiques et la prophétie en Occident, Mélanges de l'École française de Rome, Moyen-Âge, 102, 1990, p. 589596.

46. Prediche sopra i Salmi, III, éd. cit., vol. 1, p. 61 : «Dieu ne veut pas que soit prêché un temps déterminé ".

47. Cf. Prediche sopra Aggeo, I, éd. cit. p. 17 ; XIII, ibid., p. 212-213 ; Prediche sopra i Salmi, II, éd. cit., vol. 1, p. 26 ; III, ibid., p. 42 ; XXVIII, vol. 2, p. 191 ; Prediche sopra Amos e Zaccaria, V, éd. cit., vol. 1, p. 154.

48. Prediche sopra Ezechiele, VI, éd. cit., p. 69 : « [...] comme je l'ai dit plusieurs fois, c’est le Christ qui prêche et non pas moi "; cf. Prediche sopra Aggeo, I, éd. cit., p. 4 ; Prediche sopra Amos e Zaccaria, XXXIII, éd. cit., vol. 2, p. 423 ; Prediche sopra Ruth e Michea, XXVI, éd. cit., vol. 1, p. 329, et XVII, vol. 2, p. 51.

49. Ph. GuÉrin, «"La petite clé des secrets”. De la performativité... », cit., p. 28. 
totalement à Dieu, jusqu'à remplacer la troisième personne du singulier («Dieu m’a dit») par la première personne («je te dis»). Aussi peut-il déclarer : "fuori di questo luogo io ti parlo come uomo, ma quassù la va ad un altro modo " 50 .

Messager de Dieu, le prédicateur-prophète est dépositaire d'une parole qui se suffit à elle-même en vertu de son caractère sacré. Dépendante d'une puissance transcendante - Dieu - cette parole dépasse les contingences d'un discours élaboré à l'avance et travaillé selon les règles de l'éloquence. Il est significatif à cet égard que le prédicateur improvise ses sermons, en langue vulgaire, à partir de canevas qu'il a rédigés auparavant en latin et qui lui donnent des indications générales sur un sujet, mentionnent les passages des Saintes Écritures qui méritent un commentaire, ou précisent les points qui sont à approfondir en fonction de la situation du moment ${ }^{51}$. Mais si Savonarole improvise son discours, c'est aussi parce qu'il veut faire comme s'il le construisait au fur et à mesure que Dieu le lui inspire. C'est le sens des pauses oratoires qu'il réclame souvent ainsi que des dialogues qu'il imagine avoir avec son auditoire et qui s'ébauchent spontanément au cours des prêches. Ces dialogues, qui prennent la plupart du temps la forme d'un jeu de questions/réponses, ont deux fonctions. D'une part, ils servent à la fois à menacer et à rassurer l'auditoire, comme dans le sermon XX sur Job :

- O frate, credi tu però che per questo noi saremo dannati ? - Io non lo dico, leggete le Scritture e li dottori, e vedete quello che dicano. Io dico che chi non farà bene sarà condannato. ${ }^{52}$

D'autre part, ils fonctionnent comme un point d'appui justificatif lorsque Savonarole entend répondre aux protestations des Florentins. C'est le cas dans le sermon IX sur Ruth :

- O frate, e' fanciulli hanno preso ardire -. Dimmi, donde viene ch'el ti pare che gli abbino preso ardire ? Tu non vorresti che togliessino le carte e che e' levassino via li giuochi per le strade. Fanciulli, io non voglio già per niente che facciate scandalo. Se voi potete tòrre le carte loro e levare via e' giuochi senza scandalo, fatelo. ${ }^{53}$

50. Prediche sopra Aggeo, XXI, éd. cit., p. 378 : «En dehors de ce lieu je te parle comme un homme, mais en chaire il en va tout autrement ".

51. Roberto Ridolfi, Prolegomeni ed aggiunte alla "Vita di Girolamo Savonarola ", Florence, SismelEdizioni del Galluzzo, 2000, p. 143 ; G.C. Garfagninı, "Ser Lorenzo Violi e le prediche del Savonarola", cit., p. 10.

52. Prediche sopra Giobbe, XX, éd. cit., vol. 1, p. 383 : « Ô frère, tu crois donc que nous serons damnés pour cette raison ? - Je ne le dis pas, lisez les Écritures et les docteurs, et voyez ce qu'ils disent. Moi je dis que celui qui n'agira pas bien sera condamné».

53. Prediche sopra Ruth e Michea, IX, éd. cit., vol. 1, p. 267 : «- Ô frère, les enfants ont pris de l'audace -. Dis-moi, d'où te vient qu'il te semble qu'ils ont pris de l'audace? Tu ne voudrais pas qu'ils confisquent les cartes et qu'ils suppriment les jeux dans les rues. Mes enfants, je ne veux absolument pas que vous causiez du scandale. Si vous pouvez confisquer leurs cartes et supprimer les jeux sans scandale, faites-le». 
Puisque c'est Dieu qui parle en chaire par la bouche de Savonarole, la parole du prédicateur est une parole de l'immédiateté. Or, frère Jérôme écrit dans son Apologeticus de ratione poeticae artis que l'Écriture sainte est la seule à pouvoir "statim animos inclinat ad bene beateque vivendum, statimque oculos aperit ut intueantur amentque æterna " ${ }^{54}$. Elle est donc la seule à posséder le "talent oratoire " nécessaire à l'éducation des Florentins ${ }^{55}$.

L'immédiateté de la parole prophétique apparaît non seulement dans la coïncidence de la parole de Savonarole avec celle de Dieu, mais aussi dans la coïncidence de la pensée du prédicateur avec sa propre parole. Savonarole fait en effet remarquer que les prophètes « congiungono le parole di fuori con quelli di dentro " ${ }^{56}$. En cela, les prophètes s'opposent à ceux qu'il appelle les " tièdes" (tiepidi), ces mauvais religieux qui délaissent la vie intérieure au profit des " cérémonies ", parce qu'ils préferent l'apparence à la foi. En revanche, Savonarole assimile les prophètes à la fois aux "simples " et aux " apôtres ». Aux simples, parce que " quello che hanno nel cuore hanno nella lingua e nelle opere " ${ }^{57}$ et aux apôtres, parce qu'ils "predicavano semplicemente $"{ }^{58}$.

À propos des personnes simples, Savonarole observe que « questo lume della fede è più fisso in loro che la ragione nei sapienti di questo mondo " 59 . Leur simplicité est celle des pauvres ('anawim) de l'Ancien Testament, qui reconnaissent humblement leur ignorance, et qui, n'aimant que Dieu, attendent tout de lui ${ }^{60}$. Voilà pourquoi ils ne sont pas comme les " savants » - parmi lesquels se trouvent précisément les poètes et les orateurs -, qui s'appuient par orgueil sur la seule raison humaine, et que Savonarole traite ironiquement de "sages " ${ }^{61}$. Dans le Compendio di rivelazioni, Savonarole explique significativement qu'il a écarté la rhétorique qui se présentait à lui pour lui préférer « la Simplicità della fede e della sapienzia e eloquenzia delle Sacre Scritture » parce que « la filosofia e la retorica, trovate da lume

54. Apologeticus, cit., p. 255 (Nous soulignons) : «incliner sur-le-champ les âmes à vivre bien et heureusement et sur-le-champ ouvr[ir] leurs yeux pour qu'elles contemplent l'éternité et l'aiment " (La fonction de la poésie, cit., p. 146 ; nous soulignons).

55. Ibid., p. 146.

56. Prediche sopra i Salmi, III, éd. cit., vol. 1, p. 46 : "font coïncider les mots du dehors avec ce qui est dedans".

57. Prediche sopra Amos e Zaccaria, II, éd. cit., vol. 1, p. 60 : " ce que [les simples] ont dans le cœur ils l'ont sur la langue et dans leurs œuvres "; cf. Prediche sopra Ruth e Michea, V, éd. cit., vol. 1, p. 140-141.

58. Prediche sopra Aggeo, XVIII, éd. cit., p. 308 : "[...] prêchaient simplement ».

59. Prediche sopra Aggeo, II, éd. cit., p. 37 : «[...] la lumière de la foi est davantage ancrée en elles que ne l'est la raison dans les savants de ce monde».

60. Dictionnaire de la spiritualité, Paris, Beauchesne, 1960, vol. 4, p. 684-685 et p. 691.

61. Prediche sopra Aggeo, XI, éd. cit., p. 182. 
naturale della ragione, sariano troppo basse e troppo puerile appresso la maiestà di Dio e de' beati » ${ }^{62}$. La rhétorique est donc du côté de la raison naturelle, la simplicité du côté de la lumière surnaturelle. Aussi la première ne peut-elle pas convenir à la réforme de la cité, puisque pour la mener à bien les Florentins doivent avoir la foi.

La réforme de Florence dépend donc des simples, dont « la vita [...] è regola del ben vivere ", comme il est affirmé dans le sermon II sur Amos ${ }^{63}$. Savonarole s'adresse en priorité à eux parce que, tout comme Dieu, il compte parler "sempre a' semplici, non a' savi » ${ }^{64}$. Il doit dès lors rendre sa prédication accessible à ces "persone semplici [che] non [sanno] così discorrere come e' dotti " ${ }^{65}$. Pour cela il choisit de "lasc[iar] andare le sottilità», comme il le déclare de façon emblématique en annonçant qu'il va expliquer le sacrement de la communion aux enfants et aux adolescents ${ }^{66}$. Éviter les "subtilités ", c'est d'abord, comme nous l'avons vu précédemment, employer les figures de style dans une visée pédagogique et non esthétique. Éviter les "subtilités", c'est aussi éviter d'employer le latin. Certes, depuis le concile de Tours, l'Église avait recommandé que la prédication soit rendue compréhensible par l'emploi de la langue vulgaire et les ordres mendiants avaient répandu cet usage depuis le XIII ${ }^{\mathrm{e}}$ siècle ${ }^{67}$. Mais lorsque l'on sait que Mariano da Genazzano, que Laurent de Médicis utilise pour contrebalancer la prédication moralisatrice de Savonarole, n'hésite pas à prêcher parfois entièrement en latin y compris devant le peuple $e^{68}$, le fait que Savonarole traduise presque systématiquement en vulgaire les citations latines ou les accompagne d'une explication en forme de paraphrase souligne sa volonté non seulement de se mettre à la portée de l'ensemble de son auditoire, mais aussi de se démarquer des prédicateurs qui font d'après lui un usage fautif de la rhétorique.

Mais ce n'est pas tout. Savonarole affirme d'une part, que c'est des "simples » que « si vorria imparare el ben vivere " ${ }^{69}$ et d'autre part, que

62. G. Savonarola, Compendio di rivelazioni, testo volgare e latino, Dialogus de veritate prophetica, a cura di Angela Crucitti, Rome, Angelo Belardetti, 1974, p. 30 : " la Simplicité de la foi et de la sagesse et l'éloquence des Saintes Écritures »; " la philosophie et la rhétorique, trouvées par la lumière naturelle de la raison, seraient trop basses et trop puériles à côté de la majesté de Dieu et des bienheureux ».

63. Prediche sopra Amos e Zaccaria, II, éd. cit., vol. 1, p. 60 : " la vie est la règle du bien vivre ".

64. Prediche sopra Ruth e Michea, IV, éd. cit., vol. 1, p. 127 : «toujours aux simples, et non pas aux sages ».

65. Prediche sopra Aggeo, II, éd. cit., p. 37 : "personnes simples qui ne savent pas discourir comme les savants ".

66. Prediche sopra Amos e Zaccaria, XLII, éd. cit., vol. 3, p. 186 : «laisser de côté les subtilités ».

67. V. Coletti, L’éloquence de la chaire, cit., p. 53, et Lisa Bolzoni, "Oratoria e prediche ", in Letteratura italiana, a cura di Alberto Asor Rosa, Turin, Einaudi, 1984, vol. 3, t. II, p. 1042.

68. Sur frère Mariano da Genazzano, infra la note 89.

69. Prediche sopra Amos e Zaccaria, II, éd. cit., vol. 1, p. 60 : « [...] on devrait apprendre le bien vivre" ". 
«l'esempio del ben vivere è quello che convince ognuno ${ }^{70}$. Adopter la langue des "simples » revient dès lors à montrer aux Florentins l'exemple de la façon dont ils doivent se convertir. Frère Jérôme compte en effet beaucoup sur la force de l'exemplarité pour éduquer les Florentins et les conduire sur le chemin de la réforme. "Ogni nostra cognizione procede dal senso ", rappelle-t-il dans le sermon XIII sur Ézéchiel ${ }^{71}$. L'aveugle et le sourd de naissance ne connaissent ni les couleurs ni les sons parce qu'ils n'ont jamais pu en prendre connaissance. De la même façon, explique-t-il dans le sermon $V$ sur Ruth, des citoyens qui n'auraient jamais vu le péché ne pourraient que l'éviter et adopter le «bien vivre " ${ }^{72}$. Ainsi, tout comme les Florentins sont invités à imiter la sobriété vestimentaire des premiers chrétiens parce qu'elle est source de simplicité ${ }^{73}$, Savonarole s'attache à employer une langue dépouillée d'ornements rhétoriques pour que les Florentins aient sous les yeux un exemple de simplicité, car " non bisogna tanta pompa a chi vuole essere vero cristiano, bisogna simplicità " ${ }^{74}$. Savonarole ne se contente donc pas de simplifier son discours pour le rendre accessible aux «simples ", peu familiers de l'éloquence. Il entend aussi parler comme les prophètes et les apôtres, pour employer lui-même, directement, la langue des "simples ", afin qu'elle soit un modèle éducatif pour les Florentins. Sa parole est celle de la simplicité, par son contenu, mais plus encore, par sa forme même. Ce choix exclut l'usage de l'éloquence cicéronienne, comme il le dit explicitement dans le sermon IV sur Ruth :

Noi vogliamo esporre questo libro piccolino di Ruth, nel quale latentemente si demonstra la simplicità, e con essa tutta questa istoria si conforma. In questo libro parlano certe donne in certi modi, come vedrete, tutti pieni di simplicità. Dio ha fatta la Scrittura, e benché Lui parli in essa, quando vi introduce una donna a parlare, la fa parlare a uso di donna; quando uno fanciullo, gli fa dire parole da fanciullo. Dio si inclina, e fa da fanciullo con li uomini, quando el bisogna. Parla la Scrittura di Samuel, quando era fanciullo, e introducevi parole da fanciullo. Così in questo luogo parlano donne con parole amorevoli e simplici da donne, e l'una dice all'altra : "Figliuola mia e madonna mia ", come vedrai che questa istoria è molto propriamente scritta. E questo è il vero modo proprio per fare presente la cosa, come è

70. Prediche sopra Giobbe, XXXIX, éd. cit., vol. 2, p. 267 : « l'exemple du bien vivre convainc tout un chacun".

71. Prediche sopra Ezechiele, XIII, éd. cit., vol. 1, p. 170 : «toute connaissance commence par les sens ".

72. Prediche sopra Ruth e Michea, V, éd. cit., vol. 1, p. 134.

73. Voir par exemple Prediche sopra Giobbe, XX, éd. cit., vol. 1, p. 382, et G. Savonarola, De simplicitate christianae vitae, a cura di Pier Giorgio Ricci, Rome, Angelo Belardetti, 1959, p. 220-221.

74. Prediche sopra Ruth e Michea, IV, éd. cit., vol. 1, p. 125 : « celui qui veut être un vrai chrétien n’a pas besoin de tant de pompes, il a besoin de simplicité" ; cf. ibid. V, p. 134, et Prediche sopra Aggeo, VII, éd. cit., p. 118. 
(domandane questi retorici) ; ma, quando tu vuoi con vocaboli di Cicerone e eloquenti recitare una cosa di simplicità, tu la guasti. ${ }^{75}$

Savonarole aurait pu expliquer aux Florentins combien la vie des "simples » était exemplaire et les exhorter à les imiter. Mais la culture dominicaine, pour laquelle l'exemplum a une fonction d'imago agens ${ }^{76}$, lui a enseigné que l'exemplarité est bien plus efficace que les mots ${ }^{77}$. Aussi frère Jérôme affirme-t-il que connaitre la vie des saints et des martyrs et les voir revivre à travers le récit qui en est fait a beaucoup plus d'effets qu'un discours qui encouragerait à vivre comme les saints, "come etiam muove più il cibo a gustarlo che non fa ad udire recitare da altri come sia fatto el suo sapore ${ }^{78}$.

Le recours aux exempla qui indiquent les comportements à imiter ou à proscrire est caractéristique de la rhétorique pédagogique employée par les prédicateurs $^{79}$. Mais chez Savonarole, c'est la forme même du discours qui devient un exemple à imiter : celui de la simplicité. Si bien que toute l'œuvre de Savonarole - un discours de "simplicité » adressé à des personnes " simples " - est persuasive non pas parce qu' elle applique les codes de l'éloquence ni même des exempla mais parce qu'elle reflète le contenu même de son discours, elle est véritablement le contenu de ce discours. "Gli apostoli, che predicavano semplicemente, perchè avevano quella grazia del Spirito Santo, penetravano le voci loro ogni cuore ", remarque frère Jérôme ${ }^{80}$. En imitant leurs voix, il entend se faire immédiatement comprendre des Florentins et les persuader aussitôt d'agir pour la réforme de la cité.

75. Prediche sopra Ruth e Michea, IV, éd. cit., vol. 1, p. 105-106: « Nous voulons exposer ce tout petit livre de Ruth, dans lequel la simplicité est montrée de façon cachée, et toute cette histoire se conforme à elle. Dans ce livre certaines femmes parlent d'une certaine façon, comme vous le verrez, toute remplie de simplicité. Dieu a fait les Écritures, et bien qu'Il y parle, quand Il y introduit une femme pour la faire parler, Il la fait parler comme parle une femme ; quand il s'agit d'un enfant, Il lui fait dire des mots d'enfant. Dieu s'abaisse, et se comporte comme un enfant avec les hommes lorsqu'il le faut. Il fait les Écritures de Samuel enfant et Il y introduit des mots d'enfant. Semblablement dans ce livre les femmes parlent avec des mots d'amour et de simplicité propres aux femmes, et l'une dit à l'autre : " ma fille et ma dame "; comme tu le verras cette histoire est écrite de façon très appropriée. Et c'est là la véritable façon de rendre la chose présente telle qu'elle est (parles en à ces rhéteurs); mais quand tu veux parler d'une chose simple avec des mots de Cicéron et d'éloquence, tu la gâtes ".

76. Carlo Delcorno, Exemplum e letteratura tra Medioevo e Rinascimento, Bologne, il Mulino, 1989, p. 10.

77. Cf. G. Savonarole, Prediche sopra l'Esodo, a cura di Pier Giorgio Ricci, Rome, Angelo Belardetti, 1955, IV, vol. 1, p. 100.

78. Ibid. , IV, vol. 1, p. 101 : « De la même façon qu'on est davantage touché lorsqu'on goûte la nourriture soi-même que lorsqu'on entend d'autres personnes décrire sa saveur ».

79. Voir à ce propos C. DelCorno, Exemplum e letteratura, cit., en particulier p. 7-16 et p. 127-191.

80. Prediche sopra Aggeo, XVIII, éd. cit., p. 308 : "Les apôtres, qui prêchaient simplement, faisaient pénétrer leurs voix dans tous les cœurs parce qu'ils avaient la grâce de l'Esprit Saint ». 
Savonarole sait qu'un prédicateur doit donner le bon exemple parce que sans bonnes œuvres ses paroles seraient impuissantes à convertir son auditoire $^{81}$. À un premier niveau, cela correspond certes à un désir de sincérité et d'authenticité : Savonarole veut faire en sorte que son "faire " corresponde à son "dire ». Mais il y a plus. Il affirme en effet vouloir faire comme "Cristo [che] ha insegnato, e li suoi santi, co" fatti e colle opere quello che elli hanno fatto ", contrairement aux "savants " qui n'enseignent que par les " mots " ${ }^{82}$. Pourtant, il ne fait que prêcher. Jamais en effet il n'intervient sur la scène politique autrement que par sa prédication qui donne les orientations générales de la réforme, parce qu'il laisse à ses partisans le soin de s'occuper des " détails " ${ }^{83}$. Il faut alors comprendre, à un second niveau, que son ouvre, c'est sa prédication. Dans ces conditions, sa parole même devient action, comme si son " dire " était un « faire ".

Parce qu'elles proviennent de Dieu, les Saintes Écritures ont une beauté intérieure qui plaît. Parce qu'elles expriment la vérité, elles instruisent. Or, il est une autre fonction de l'éloquence qu'elles remplissent parfaitement aux yeux de Savonarole : « nec umquam relinquit in cortice litteræ, sed ad finem intentum fortiter perducit ${ }^{84}$. Autrement dit, elles émeuvent, c'està-dire qu'elles font agir, et qui plus est, elles font agir dans le respect de la volonté divine.

Dans le sermon VIII sur Aggée, frère Jérôme exhorte les Florentins à venir assister à son prêche en ces termes :

Vivete adunque da cristiani e venite alle predicazioni, dove s'impara el ben vivere cristiano ; chi non vuole udire el verbo di Dio fa in prima ingiurie a Dio e, secundario, dà scandalo nel popolo. E però andate alle prediche ad imparare, e saperete meglio reggervi secondo Dio. 85

81. Ibid., XXII, p. 400-401.

82. Ibid., V, p. $85:$ « [...] le Christ et ses saints ont enseigné par les faits et les œuvres ce qu'ils ont fait "; cf. Prediche sopra Amos e Zaccaria, VII, éd. cit., vol. 1, p. 208.

83. Cf. Prediche sopra Amos e Zaccaria, XIX, éd. cit., vol. 2, p. 39 ; Prediche sopra Ruth e Michea, XIII, éd. cit., vol. 1, p. 411-412 et XIX, vol. 2, p. 122 ; I tre processi apocrifi del Savonarola, a cura di Pasquale Villari, in Pasquale Villari, La storia di Girolamo Savonarola e de' suoi tempi, Florence, Le Monnier, 1930, vol. 2, appendice, p. clvij. Les partisans de Savonarole s'inspirent effectivement de ses sermons et s'entretiennent parfois directement avec lui, mais les détails des décisions politiques sont laissés entre les mains des principaux piagnoni membres du grand conseil. Voir à ce propos Lorenzo Polizzotto, The Elect Nation. The Savonarolan Movement in Florence 1494-1545, Oxford, Clarendon Press, 1994, p. 21, et Nicolai Rubinstein, «Politics and Constitution in Florence at the end of the Fifteenth Century ", in Italian Renaissance Studies. A tribute to the late Cecilia M. Ady, éd. E.F. Jacob, Londres, Faber and Faber, 1960, p. 166-167.

84. Apologeticus, cit., p. 255 : « elles n'en reste[nt] pas à l'écorce de la lettre mais mène[nt] irrésistiblement à la fin visée " (La fonction de la poésie, cit., p. 146).

85. Prediche sopra Aggeo, VIII, éd. cit., p. 135: «Vivez donc en chrétiens et venez à la prédication où 
Nous avons vu qu'apprendre le "bien vivre " et se " gouverner selon Dieu " sont les conditions préalables et nécessaires à la réforme politique de la cité. Par conséquent, si les Florentins refusent d'écouter Dieu, ils refusent aussi de réformer leur ville. Inversement, s'ils l'écoutent, ils agissent pour la réforme. Si bien que lorsque Savonarole affirme que seule la formation que les Florentins reçoivent en écoutant ses sermons peut faire d'eux de «bons » citoyens et leur permettre d'acquérir la sagesse nécessaire pour réformer Florence, il fait de ses sermons l'instrument même de cette réforme ; une réforme qui n'est pas seulement religieuse et morale, mais qui est aussi politique, inscrite dans le temps humain de la cité. Prêcher revient alors à mettre en place la réforme. Ce n'est d'ailleurs pas un hasard si les lois des 22 et 23 décembre 1494 instituant le grand conseil et le conseil des quatre-vingts suivent les sermons des 7 et 14 décembre où Savonarole avait précisément évoqué le modèle institutionnel vénitien ${ }^{86}$. Mieux encore, le 21 décembre il espérait que la réforme aboutirait le jour même : "spero che forse oggi si farà ; se non, almanco innanzi la vigilia di Natale $" 87$.

De plus, lorsque Savonarole emploie une langue "simple " comme celle des apôtres et des prophètes afin de montrer l'exemple de la «simplicité " à son auditoire, il introduit dans la cité une "nouvelle coutume »-le retour de l'âge d'or des premiers chrétiens - qui chasse le "vieil usage » - le temps des prédicateurs qui parlaient en chaire comme des poètes. Autrement dit, il fait exactement ce qu'il recommande aux Florentins de faire, et ce changement est suscité par l'emploi nécessaire d'une parole nouvelle.

Cerretani soulignera d'ailleurs significativement le caractère inédit de la parole savonarolienne, qui dira que le prédicateur

introduxe quasi nuovo modo di pronuntiare il verbo d'Iddio, c[i]oè a l'apostolescha sanza dividere el sermone, non proponendo quistione, fugendo el chantare, gl'ornamenti d'eloquentie, solo il suo fine era exporre qualcosa del vecchio testamento et introdurre la semplicità della primitiva chiesa. ${ }^{88}$

\footnotetext{
on apprend le bien vivre chrétien ; celui qui ne veut pas écouter le verbe de Dieu fait d'abord injure à Dieu et provoque ensuite du scandale dans le peuple. Et c'est pourquoi allez à la prédication pour apprendre, et vous saurez mieux vous gouverner selon Dieu ».

86. Pour une mise au point sur les événements de cette période, voir Guidubaldo Guidi, Ciò che accadde al tempo della Signoria di novembre dicembre in Firenze l'anno 1494, Florence, Arnaud, 1988.

87. Prediche sopra Aggeo, XIX, éd. cit., p. 343 : «J'espère qu'on la fera peut-être aujourd'hui ; sinon, au moins avant la veille de Noël ".

88. Bartolomeo Cerretani, Storia fiorentina, a cura di Giuliana Berti, Florence, Olschki, 1994 , p. $184:$ « [...] il introduisit presque une nouvelle façon de prononcer le verbe de Dieu, c'est-à-dire comme les apôtres, sans diviser son sermon, sans proposer de questionnement alambiqué, fuyant le chant, les ornements d'éloquence. Son seul but était d'exposer des passages de l'Ancien Testament et d'introduire la simplicité de l'Église primitive ».
} 
Cette façon de prêcher apparaît «nouvelle » à Cerretani parce que le public florentin qui écoute frère Jérôme est effectivement habitué à d'autres formes de sermons. Tandis que ses condisciples dominicains privilégient les syllogismes et le raisonnement scolastique, les franciscains adoptent une langue parsemée d'anecdotes populaires. C'est toutefois la comparaison avec le frère augustinien Mariano da Genazzano qui est la plus frappante ${ }^{89}$.

Au début du XVI ${ }^{\mathrm{e}}$ siècle, Niccolò Valori écrira à propos de frère Mariano qu'il était

famoso non solo per le sacre lettere, ma per la soavità del predicare et per la eloquenza con la quale tanto poteva, che nelle sue concioni et prediche non solo i popoli lo seguitavano, ma i bene letterati. ${ }^{90}$

Quelques années plus tôt, dans sa dédicace des Miscellanea à Laurent de Médicis, Ange Politien expliquait semblablement que Mariano maîtrisait parfaitement la théologie et qu'en même temps « il ne mépris[ait] pas les beautés de la poésie ». De cette association de la doctrine religieuse et de la culture profane découle une éloquence qui sait instruire, émouvoir et plaire. Aussi Mariano peut-il apparaître à Jean Pic de la Mirandole comme " le modèle de la prédication humaniste abreuvée à la source cicéronienne et quintilienne ", comme l'observe Marc Deramaix ${ }^{91}$. Dès lors, en 1490, les lettrés qui apprécient Mariano surnomment Savonarole « il predicatore dei disperati " ${ }^{92}$. Ce à quoi le dominicain répond, comme il le rappellera cinq ans plus tard dans le sermon XXII sur les Psaumes, que les « désespérés » sont justement ses détracteurs qui au lieu de penser à se convertir et à participer à la réforme de la cité voulue par Dieu ne pensent qu’à "difendere el Morgante e chi faceva sonetti contro alla fede $"{ }^{93}$.

89. Pour ce développement, j'utilise l'étude suivante : Marc DeramaIx, "Consumatum est. Rhétorique et prophétie dans un sermon de Mariano da Genazzano contre Savonarole ", in Savonarole. Enjeux, débats, questions, cit., p. 173-197. Sur Mariano da Genazzano, on pourra également consulter David Gutierrez o.s.a., "Testi e note sur Mariano da Genazzano », in Analecta Augustiniana, 32, 1969, p. 117-204, et John W. O’Malley, Praise and Blame in Renaissance Rome. Rhetoric, Doctrine and Reform in the Sacred Orators of the Papal Court, c. 1450-1521, Durham, Duke University Press, 1979.

90. Niccolò Valori, Vita di Lorenzo il Magnifico, introduzione di Angela Dillon Bussi, note di Anna Rita Fantoni, Palerme, Sellerio, 1992, p. 82: «fameux non seulement par sa connaissance des lettres sacrées mais encore pour la douceur de son prêche et pour son éloquence qui lui donnait tant de pouvoir que, dans ses harangues et dans ses prêches, non seulement le peuple le suivait mais aussi les lettrés "; j'emprunte la traduction de Marc Deramaix.

91. M. Deramaix, "Consumatum est. Rhétorique et prophétie », cit., p. 177.

92. Roberto Ridolfi, Vita di Girolamo Savonarola, sesta edizione ancora riveduta, Florence, Sansoni, 1981 , p. 56 : «Le prédicateur des désespérés ».

93. Prediche sopra i Salmi, XXII, éd. cit., vol. 2, p. 80 : " [...] défendre le Morgante et ceux qui faisaient des sonnets contre la foi ». 
Domenico Benivieni avait fait remarquer à Savonarole qu'il était perdant lorsque les Florentins le comparaient à Mariano da Genazzano. En effet, expliquait Benivieni, tandis que frère Mariano agrémente ses sermons d'ornements poétiques, frère Jérôme prêche sans prendre garde à la beauté des paroles ni à la façon de les prononcer et de les mettre en scène. Mais voici ce que Savonarole lui avait rétorqué :

egli è la verità, ma voi avete a intendere che questa facondia ed eleganzia e modo di predicare del padre fra Mariano hanno a perder di grazia assai, e la semplicità del predicar nostro ha da essere esaltata. ${ }^{94}$

Cette façon de parier sur l'avenir correspond à une revendication de la part de Savonarole de " la performativité de son discours », selon l'analyse de Philippe Guérin ${ }^{95}$. Ce discours doit modifier la cité florentine en y introduisant une " nouvelle coutume " - l'âge d'or des premiers chrétiens d'une part, la prédication semblable à celle des apôtres d'autre part. Et si ce discours cherche à introduire une "nouvelle coutume » à Florence, c'est parce que

secondo la diversità de' tempi [...] mutarsi e trovarsi novi modi di vivere non solamente non è inconveniente, ma è cosa necessaria. ${ }^{96}$

Les temps ont justement changé depuis que Charles VIII a franchi les Alpes le $1^{\text {er }}$ septembre 1494. Ayant fait des promesses inconsidérées au souverain français, Pierre de Médicis a été chassé de Florence, ce qui a provoqué la chute du régime fondé par son arrière-grand-père. Changement qui donna du même coup à Savonarole la possibilité d'intervenir dans la vie politique pour proposer une réforme des institutions.

Cette intervention lui vaut rapidement des ennemis. Savonarole sait et déclare qu'il doit mener une véritable guerre contre ceux qu'il appelle les cattivi, ces mauvais citoyens qui ne veulent pas d'une "nouvelle coutume " pour Florence. Jean-Louis Fournel et Jean-Claude Zancarini ont montré comment Savonarole joue en permanence sur l'équilibre entre la violence des mots (la certitude de dire vrai qui peut conduire au martyre) et la violence des armes (destinée à éliminer physiquement ses adversaires) ${ }^{97}$. Mais, comme ils le soulignent aussi, Savonarole ne peut se résoudre en

94. Cité par R. Ridolfi, Vita di Girolamo Savonarola, cit., p. 52 : «C'est vrai, mais vous devez comprendre que la faconde, l'élégance et la façon de prêcher du frère Mariano vont perdre beaucoup de leur grâce, alors que la simplicité de notre prêche sera exaltée ».

95. Ph. Guerin, " "La petite clé des secrets". De la performativité... ", cit., p. 19-20.

96. Prediche sopra Aggeo, XV, éd. cit., p. $249:$ : [...] selon la diversité des temps [...] changer et trouver de nouvelles façons de vivre est non seulement une chose convenable, mais c'est aussi une chose nécessaire "; cf. ibid., XVI, p. 263.

97. J.-L. Fournel - J.-C. ZANCARINI, « La question de l'ennemi : "faire la guerre avec la paix dans le coeur" ", in La politique de l'expérience, cit., p. 55-73. 
définitive à préconiser l'emploi de la violence et des armes, ces " moyens extraordinaires " que Machiavel indiquera au contraire comme nécessaires pour réformer la cité "d'un seul coup ", ad un tratto ${ }^{98}$. Le prédicateur, refusant de " pigliare istraordinaria autorità " ${ }^{99}$, dira encore Machiavel, explique en ces termes son choix de régler les conflits sans violence : « a tutti quelli che avessino cercato di farmi male, non crediate che io voglia male nessuno $"{ }^{100}$.

Ce refus de la violence des armes est d'abord dû au fait que Savonarole n'envisage pas la réforme de la cité dans une perspective de rupture. Ainsi observe-t-il que

a volere mettere una nuova consuetudine contro l'usanza vecchia, non si può cos̀̀ in un tratto intromettere : ma bisogna andare pian piano. Pertanto, volendo noi col nostro predicare introdurre nuova consuetudine e demostrare la renovazione che si debba fare per contraria consuetudine, ci bisogna andare pian piano e a poco a poco. ${ }^{101}$

Savonarole cherche à construire une nouvelle cité à partir de l'ordre ancien. Jamais il ne supprime les coutumes des habitants, mais il les transforme parce que, selon sa propre formule, "io non cerco di guastare, ma di racconciare quello che fusse guasto ${ }^{102}$.

Pour ce faire, il compte sur le pouvoir de la parole pour éduquer les Florentins et les inciter au renouveau : " ti bisogna con le parole e la dottrina e colle esortazioni e riprensioni correggere e ritenere chi tu hai in cura " ${ }^{103}$. Les recommandations qu'il fait aux fanciulli, lorsqu'il leur demande de corriger fraternellement leurs aînés, vont dans ce sens :

Io intendo che si giuoca. [...] Voi fanciulli, se lo intendete, accusategli, ma non andate a volere entrare nelle case per forza, ché non voglio che facciate scandalo. ${ }^{104}$

98. Discorsi sopra la prima deca di Tito Livio I, $18:$ : [...] allo straordinario, come è alla violenza e all'armi " (Niccolò Machiavelli, Discorsi sopra la prima deca di Tito Livio, introduzione di Gennaro Sasso, note di Giorgio Inglese, Milan, Rizzoli, 1996).

99. Ibid., III, $3:$ : [...] prendre une autorité extraordinaire".

100. Prediche sopra Ruth e Michea, XIII, éd. cit., vol. 1, p. 411 : « [...] à tous ceux qui auraient cherché à me faire du mal, ne croyez pas que je veuille leur faire du mal ».

101. Prediche sopra Aggeo, IX, éd. cit., p. 143 : "Quand on veut mettre une nouvelle coutume à la place du vieil usage, on ne peut pas l'introduire tout d'un coup ; mais il faut aller tout doucement. C'est pourquoi, puisque nous voulons par notre prêche introduire une nouvelle coutume et montrer le renouveau qui doit se faire contre une coutume contraire, il faut aller tout doucement et petit à petit ».

102. Prediche sopra Giobbe, XX, éd. cit., vol. 1, p. 382 : «je ne cherche pas à gâter, mais à réparer ce qui a été gâté ".

103. Prediche sopra i Salmi, XVII, éd. cit., vol. 1, p. 284-285 : « [...] il faut corriger et contrôler ceux dont tu as la charge par les mots et le savoir et par les exhortations et les reproches».

104. Prediche sopra Amos e Zaccaria, XVII, éd. cit., vol. 1, p. 427-428 : «J'entends que l'on joue. [...] Vous, enfants, si vous entendez [les joueurs], accusez-les, mais ne rentrez pas de force dans les maisons, car je ne veux pas que vous fassiez du scandale "; voir aussi Prediche sopra Ruth e Michea, IX, éd. cit., vol. 1, p. 267. 
Les fanciulli doivent donc inciter les « mauvais citoyens " à renoncer à leurs vices en les accusant publiquement et en les exhortant, par le harcèlement, à changer de vie. Ils ne doivent pas exercer de contrainte physique contre les pécheurs qu'ils pourchassent, mais les persuader, par la parole, à se convertir. Comme les pédagogues humanistes, Savonarole est convaincu que l'exemple et la persuasion sont plus efficaces que la force des châtiments corporels pour faire émerger les bonnes dispositions naturelles propres à l'individu ${ }^{105}$. Mais ce point de vue va bien plus loin qu'une simple prise de position sur des méthodes éducatives.

En donnant un tel pouvoir effectif et performant à la parole, Savonarole prend modèle sur le Christ et ses apôtres qui vainquirent « certo non con arme, non con cavagli, ma con la lingua sola " ${ }^{106}$. Machiavel fera de ce refus d'employer les armes la cause de l'échec et de la chute de Savonarole, qualifiant le prédicateur de "prophète désarmé " ${ }^{107}$. Pourtant, le combat est bien présent tout au long de sa prédication. Mais c'est un combat de mots. Frère Jérôme possède, comme l'ont formulé Jean-Louis Fournel et Jean-Claude Zancarini, "l'arme de la parole " ${ }^{108}$. Certes, Savonarole encourage les Florentins, dès le 16 novembre 1494, " a volere essere cristiano per amore e non per forza " ${ }^{109}$. Autrement dit, le prédicateur n'exercera aucune contrainte physique sur les Florentins, dont la conversion doit venir d'eux-mêmes. Mais cette conversion ne sera possible que parce que Savonarole les aura persuadés de sa nécessité en utilisant une arme

105. Nombreux sont en effet les humanistes qui dénoncent l'emploi des châtiments corporels. Voir par exemple Leon Battista AlberTi, I libri della famiglia, a cura di Ruggiero Romano e Alberto Tenenti, Turin, Einaudi, 1969, p. 70-71 ; Matteo Palmieri, Vita civile, a cura di Gino Belloni, Florence, Sansoni, 1982, p. 35 ; Maffeo Veggio, De educatione liberorum et claris eorum moribus in Eugenio Garin, Educazione umanistica, Bari, Laterza, 1949, p. 52-53. À notre connaissance, Savonarole ne se prononce pas sur l'emploi des punitions physiques. Mais sous bénéfice d'inventaire, le seul passage où il fait allusion à des coups concerne des vieillards, dont il dit qu'il faudrait les frapper jusqu'à ce qu'ils renoncent à leurs vices (Prediche sopra Ruth e Michea, V, éd. cit., vol. 1, p. 135). En outre, il s'agit plus là d'une expiation des péchés que d'un processus éducatif. On peut également noter que le médecin Michele Savonarola, le grand-père du prédicateur, recommandait aux maîtres de solliciter leurs élèves " cum poche bote e molte loxenge " («avec peu de coups et bien des louanges ") et ajoutait que si le maître est " prudent ", il se fait davantage obéir et craindre pour le respect qu'il inspire que s'il frappait ; cf. Il trattato ginecologico-pediatrico in volgare di Michele Savonarola, a cura di Luigi Belloni, Per il XLII Congresso della società italiana di ostetrica e ginecologia, Milano, 16-19 ottobre 1952.

106. Prediche sopra Amos e Zaccaria, VII, éd. cit., vol. 1, p. 196 : «Non pas certes avec les armes, non pas avec les chevaux, mais seulement avec la parole ", .

107. N. Machiavel, De Principatibus. Le Prince, traduction et commentaire de J.-L. Fournel et J.-C. Zancarini, texte italien établi par Giorgio Inglese, Paris, PUF, 2000, VI ; N. Machiavelli, Discorsi, cit., I, 9 ; I, 16 ; III, 4 et III, 30.

108. Il s'agit du titre donné à la préface de l'ouvrage J. Savonarole, Sermons, écrits politiques et pièces du procès, textes traduits, présentés et annotés par J.-L. Fournel et J.-C. Zancarini, Paris, Seuil, 1993, p. 140 .

109. Prediche sopra Aggeo, V, éd. cit., p. 86 : «[...] à vouloir être chrétiens par amour et non pas par la force" . 
efficace : sa langue. Cette langue qui est un "flagello di fune ", semblable à celui employé par Jésus pour chasser les marchands du Temple, « el quale denunzia che egli verrà el Signore e che ognuno si converta " ${ }^{110}$, et " la spada del verbo di Dio [che] tagli[a] via tutti e' vizii del popolo $"{ }^{111}$.

Par conséquent, Savonarole ne veut pas de la violence des armes, mais il envisage en revanche l'art de persuader comme un véritable combat à mener par la parole, employée comme une arme. De ce point de vue, il est révélateur qu'il considère que "el flagello di fune nella città di Firenze sono e' fanciulli " ${ }^{112}$. Ces enfants à qui il défend d'exercer une quelconque violence, mais qu'il encourage d'un autre côté à chasser les " mauvais » citoyens de la cité. Par la parole il désigne des soldats, par la parole ses soldats devront combattre.

Un des biographes partisans de Savonarole, le pseudo-Burlamacchi, avait bien perçu toute l'ambivalence de ce soldat pacifique, qui remarquait que « le parole sue erano sagitte et faccelline accese negli quori nostri " ${ }^{113}$. Savonarole combat et apaise, menace et fait espérer, et ce, uniquement par la parole : il sait se montrer persuasif. Instruments politiques, ses sermons sont alors autant d'actes en faveur de la réforme de la cité. C'est pourquoi ses adversaires ont tant cherché à le faire taire, jusqu’à le condamner à mort.

Pour Savonarole, la lutte contre les cattivi et pour le renouvellement de Florence représente la mission sacrée qui selon lui lui vient de Dieu. Mais ses détracteurs considèrent justement que cette mission, qui consiste à se battre avec la parole pour que la cité adopte une "nouvelle coutume ", perturbe l'ordre de la péninsule. Savonarole donne une réponse apparemment contradictoire à cette accusation.

Colui dice : - O frate, el tuo dire è quel che conturba la Italia -. O stolto ! Se il mio dire conturba la Italia, che vuol dire che già quattro o cinque anni e anche più io dicevo queste cose medesime e non conturbava la Italia ? Perché e’ non erano ancora

110. Prediche sopra Amos e Zaccaria, XXVII, éd. cit., vol. 2, p. $260:$ « [...] le fouet de cordes [...] qui annonce que le Seigneur viendra et que chacun doit se convertir».

111. Prediche sopra Giobbe, XXI, éd. cit., vol. 1, p. 399 : « [... l l'épée du verbe de Dieu [qui] élimin[e] tous les vices du peuple»".

112. Prediche sopra Amos e Zaccaria, XXVII, éd. cit., vol. 2, p. 260 : «Le fouet de cordes dans la cité de Florence ce sont les enfants".

113. La vita del beato Ieronimo Savonarola scritta da un anonimo del secolo XVI e già attribuita a fra Pacifico Burlamacchi pubblicata secondo il codice ginoriano, a cura del Principe Piero Ginori Conti, Florence, Olschki, 1937, p. 91 : «Ses paroles étaient des flèches et des petits flambeaux allumés dans nos cœurs ». Il est intéressant de noter à ce propos que Savonarole avait lui-même fait appel aux paroles du Seigneur en les comparant à des flèches : « Sagittae tuae acutae: vieni, Signore, con le tue sagitte, idest con le tue parole e con le tue sentenzie che penetrano e' cuori " (Prediche sopra Amos e Zaccaria, éd. cit., XXXVIII, vol. 2, p. 115). 
venute gente fuora di Italia a conturbar la Italia. Dunque queste gente sono la conturbazione della Italia. Se dunque el mio dire in questo modo conturba la Italia, séguita che il mio dire sia fare. Essendo questo proprietà divina, bisogna dire o che io sia Dio o che le mie parole procedino da Dio. Sed sic est, che io non sono Dio, ma uomo ; dunque le mie parole procedono da Dio. ${ }^{114}$

Ce que Philippe Guérin considère comme un paralogisme (le deuxième " donc » contredirait le premier) ${ }^{115}$ peut en fait être élucidé par l'importance que Savonarole confere à la " diversité des temps ». Si le discours du prédicateur a troublé l'Italie, c'est parce qu'il agissait pour la réforme, il était action. Mais s'il était action, s'il devait être action, c'est parce que les circonstances, les «temps » avaient changé. Dès lors, les Florentins ont été " bouleversés " par une prédication qui correspondait à la " diversité des temps » et qui les renvoyait, comme le souligne Savonarole, à la venue de " gens étrangers".

Or, ces « gens étrangers » ont introduit dans la guerre la rapidité, « spacciando ed acquistando in meno tempo uno regno che prima non si faceva una villa ; le espugnazione delle città velocissime e condotte a fine non in mesi ma in dì ed ore ", expliquera Francesco Guicciardini dans ses Storie fiorentine ${ }^{116}$. Pour s'adapter à cet état d'urgence permanent, Savonarole ne prend pas le temps d'élaborer ses sermons à l'avance. Il les conçoit au jour le jour, en pliant leur contenu aux exigences du moment présent, en faisant des recommandations pour orienter les décisions politiques, en orchestrant les processions qui auront lieu dans la cité, en éduquant les fanciulli pour qu'ils guident leurs aînés dans le droit chemin. Et pour que, de leur côté, les Florentins puissent agir rapidement, il emploie une langue immédiatement accessible et compréhensible. Ses sermons sont en langue vulgaire pour que tous le comprennent. Les figures de style telles que la métaphore et l'allégorie sont explicitées pour que son auditoire puisse en tirer profit au plus vite. Le langage employé est celui de la "simplicité » pour que les «simples", qui ne connaissent pas l'art de la rhétorique, puissent y avoir accès mais aussi pour que tous les Florentins bénéficient d'un

114. Prediche sopra Amos e Zaccaria, I, éd. cit., vol. 1, p. 46 : "Celui-là dit : - Ô frère, c'est ton dire qui bouleverse l'Italie. - Ô sôt ! si mon dire bouleverse l'Italie, que signifie le fait qu'il y a quatre ou cinq ans et même davantage, je disais déjà ces mêmes choses et je ne bouleversais pas l'Italie ? Car il n'était encore venu personne de l'étranger pour bouleverser l'Italie. Ce sont donc ces gens qui sont le bouleversement de l'Italie. Si donc mon dire bouleverse de cette façon l'Italie, il s'ensuit que mon dire est un faire. Comme c'est là une propriété divine, il faut dire ou que je suis Dieu ou que mes paroles procèdent de Dieu. Mais comme je ne suis pas Dieu, mais homme, donc mes paroles procèdent de Dieu ».

115. Ph. Guerin, " "La petite clé des secrets". De la performativité... ", cit., p. 19.

116. Francesco Guicciardini, Storie fiorentine, a cura di Alessandro Montevecchi, Milan, Rizzoli, 1988 , p. $197:$ : [...] on perdait et on acquérait des royaumes en moins de temps qu'il n'en fallait autrefois pour un village ; on perdait les villes très vite et il ne fallait plus des mois mais quelques jours, quelques heures, pour en venir à bout». 
modèle de simplicité à imiter. Au total, l'éloquence savonarolienne sera l'éloquence des Saintes Écritures, dont on a vu qu'elle fait agir "sur le champ ». Là, se trouve l'art de persuader de Savonarole, qui veut faire agir immédiatement, tout comme lui-même agit immédiatement en prenant la parole. Sa parole est alors une véritable arme, au service de l'action.

Outre la rapidité, les guerres d'Italie suscitent un sentiment d'insécurité face à un monde chaotique dominé par le désordre et l'imprévisible, où les alliances se modifient sans cesse. Après le passage des Français, l'heure n'est plus à la rigidité de la dispositio et aux stratégies rhétoriques de persuasion, mais à l'improvisation au gré des événements, qui saura se conformer au désordre pour tenter, justement, de trouver de nouveaux instruments de compréhension de la réalité et de nouvelles solutions politiques. L'art de persuader consistera alors dans l'art de savoir s'adapter à la " diversité des temps ».

Par ailleurs, lorsque Savonarole observe que « [s] on dire est un faire », il rappelle que ce n'est pas lui qui parle mais Dieu. Or, parce que le prédicateur est un homme de Dieu, sa parole est elle-même persuasion, elle " émeut », elle fait agir et surtout elle agit : son « dire » est un « faire ». Et puisque la réforme religieuse est le fondement de la réforme politique, la prédication savonarolienne peut être considérée comme l'arme d'un discours politique. Mais en nouant de cette façon religion, morale et politique, Savonarole rompt avec la conception florentine traditionnelle qui défendait au contraire l'autonomie de la politique et de la morale - Côme de Médicis n'avait-il pas l'habitude de dire que "gli stati non si tenevono co' paternostri in mano " 117 ? Au début du Xvi siècle, Machiavel et Francesco Guicciardini réaffirmeront qu'il est impossible de mettre sur le même plan l'action politique et les règles de la morale religieuse. Cependant, ils auront hérité de la prédication savonarolienne un point autour duquel s'articulera leur réflexion politique : le discours politique doit tenir compte de la conjoncture, de la " condition des temps" (condizione dè tempi) pour Guicciardini et de la " qualité des temps » (qualità de' tempi) pour Machiavel ${ }^{118}$.

Si l'on en croit Cinozzi, qui était pourtant un admirateur de Savonarole, l'auditoire de ce dernier à San Lorenzo pendant le carême 1484 se rédui-

117. Istorie fiorentine, VII, $6:$ " on ne gouverne pas avec des patenôtres " (N. Machiavelli, Tutte le Opere, a cura di Mario Martelli, Florence, Sansoni, 1993, p. 797).

118. Sur l'importance de la conjoncture dans la pensée politique florentine au début du Xvi ${ }^{\mathrm{e}}$ siècle, voir J.-L. Fournel \& J.-C. ZanCARIni, La politique de l'expérience, cit. 
sait à moins de vingt-cinq personnes, "perché né in gesti né in pronunzia satisfece quasi a nessuno " ${ }^{119}$. En 1498, le prédicateur lui-même reconnaît que dix ans auparavant " era in fastidio ad ogni uomo il mio predicare " parce qu'il n'avait " nè voce nè petto nè modo di predicare " ${ }^{120}$. En revanche, dans les années 1494-1498, alors qu'il a certes pris l'habitude de s'exprimer en chaire, mais qu'il ne prête pas plus attention à son élocution, Savonarole attire des foules d'auditeurs. Le pseudo-Burlamacchi rapporte ainsi que

[...] si levavono li suoi devoti in gran numero a mezza notte per pigliare il luogo alla predica, et venendo alla porta della chiesa, all'aria stavano più hore, a' freddi, a sottilità dell'aria, sopra le pietre et marmi coi piedi. ${ }^{11}$

Son témoignage est confirmé par celui de Piero Vaglienti. Appartenant au camp des ennemis de Savonarole, il mentionne semblablement que " a mezza notte si levavano le donne e li uomini per andare in Santa Maria del Fiore a pigliare e luoghi ${ }^{122}$.

Une des raisons du succès de Savonarole tient sans doute au fait que les contemporains ont lu certains événements comme la réalisation des prédictions qu'il avait formulées. Simone Filipepi par exemple affirme que "molte cose predisse in quei primi tempi, che si verificarono, come la morte d'Innocentio, di Lorenzo de'Medici, et altri accidenti " 123. Autrement dit, tout se passe comme si la parole de Savonarole avait le pouvoir de déclencher l'action, mieux, qu'elle était action. Significativement, dans sa Storia fiorentina, Cerretani parle d'une façon concrète de la construction de l'arche de pénitence imaginée par Savonarole pour sauver les Florentins repentants :

$[\ldots]$ cominciò a predichare et cominciò a edifichare una archa, come fe' nel testamento vechio Noè, et in ogni predicha edifichava et commetteva quatro assi chon

119. Estratto d'una epistola fratris Placidi de Cinozis Ordinis Praedicatorum S. Marci de Florentina, De vita et moribus reverendi patris fratris Hieronimi Savonarole de Ferraria, fratri Iacobo Siculo, eiusdem Ordinis vicarius generalis (sic), post mortem dicti Prophete, in Scelta di prediche e scritti di fra Girolamo Savonarola, cit., p. 11 : "parce que ni ses gestes ni sa prononciation ne satisfaisaient presque personne".

120. Prediche sopra l'Esodo, II, éd. cit., vol. 1, p. 50 : " ma prédication déplaisait à chacun "; " ni la voix ni le souffle ni la façon de prêcher ».

121. La vita del beato Ieronimo Savonarola, cit., p. 90 : «[...] ses fidèles se levaient en grand nombre à minuit pour avoir leur place à la prédication, et ils venaient aux portes de l'église, ils restaient plusieurs heures dehors, exposés au froid, à l'air perçant, les pieds sur les pierres et le marbre ».

122. Piero Vaglienti, Storia dei suoi tempi 1492-1514, a cura di G. Berti \& Michele Luzzati \& Ezio Tongiorgi, Pise, Nistri-Lischi e Pacini Editori, 1982, p. 33 : "les femmes et les hommes se levaient à minuit pour aller à Santa Maria del Fiore réserver leur place ".

123. Estratto della Cronaca di Simone Filipepi nuovamente scoperto nell'Archivio Vaticano, in Scelta di prediche e scritti di fra Girolamo Savonarola, cit., p. 475 : "de nombreuses choses qu'il prédit en ces premiers temps se vérifièrent comme la mort d'Innocent [VIII], de Laurent de Médicis, et d'autres accidents". 
espositioni mirabili, proponenddo che presto verrebbe il diluvio c[i] oè soldati et primcipi che pigl[i] erebbono le ciptà le forteze solo cholla presentia, et che Italia era spaciata et che la non haveva remedio, et che tutto questo diceva per partte d'Iddio. ${ }^{124}$

Le prédicateur est ainsi présenté comme un charpentier dont l'outil de travail serait la parole. Or, c'est bien cette "parole agissante ", comme l'écrit Philippe Guérin ${ }^{125}$, qui va caractériser la pensée politique florentine au début du XVI ${ }^{e}$ siècle. Les analyses de la situation présente de la ville et l'écriture de son histoire se multiplient parce que leurs auteurs comptent que leurs textes auront des effets dans la cité. Écrire, c'est alors pour de nombreux Florentins prendre part au débat sur la forme que doit avoir leur gouvernement, d'autant qu'ils participent souvent eux-mêmes à la gestion des affaires publiques, comme c'est le cas pour Machiavel et Francesco Guicciardini. L'écriture permet de "faire le point " (fermare il punto), comme aime à le dire Guicciardini, afin de mieux comprendre la situation présente pour pouvoir ensuite trouver les solutions adaptées à la conjoncture et définir la meilleure façon d'agir.

Dès lors, il semblerait que Savonarole ait non seulement inauguré en 1494 un débat politique qui se prolongera jusqu'à l'instauration du principat en 1537 mais qu'il ait aussi contribué à forger une nouvelle parole politique, qui serait tout entière vouée à l'action dans le temps présent de la cité. Dès le début des guerres d'Italie, il a compris d'une part, que l'état d'urgence exigeait des décisions rapides et non des raisonnements rhétoriques dont les Florentins étaient jusqu'alors friands, eux qui avaient l'habitude de compter sur le "bienfait du temps" (benefizio del tempo) pour résoudre les conflits; d'autre part, que la "diversité des temps » nécessitait de nouveaux outils pour diriger la cité. Pour ces deux raisons, il lui fallait trouver un nouvel art de persuader pour que le discours favorise une action immédiate.

124. B. Cerretani, Storia fiorentina, cit., p. 193 : « [...] il commença à édifier une arche, comme le fit Noé dans l'Ancien Testament, et dans chaque sermon il édifiait et assemblait quatre planches avec des propos étonnants, en disant que rapidement viendrait le déluge c'est-à-dire des soldats et des princes qui prendraient les villes et les forteresses par leur seule présence, et que l'Italie était perdue et qu'elle n'avait pas de remède, et qu'il disait tout cela de la part de Dieu ".

125. Ph. GuÉrIn, " "La petite clé des secrets”. De la performativité... », cit., p. 19. 